\title{
Tapered fiber sensor in the near infrared wavelength
}

\author{
JIANG-YAN LI* \\ Departerment of Information Engineering, Binzhou University, Binzhou, Shandong Province, China
}

\begin{abstract}
Simulated transmission spectra for tapered fibers with no taper, one taper and two tapers in the near infrared wavelength range, calculated by Finite-Difference-Time-Domain method are currently presented. Transmission peak positions tend to shift to the shorter wavelength when the taper deformation is added to the fiber or the taper width gets narrower. The thickness sensitivity for the tapered structures with different taper thicknesses is about $2.28 \mathrm{e}-3 \mathrm{~nm} \cdot \mu \mathrm{m}^{-1}$. There is an interference structure in the electric field distribution images, which reveals in the fiber structures. The transmission spectra for the fiber without taper, one taper and two-tapered structures were simulated in near infrared wavelength by FDTD. The transmission spectra for tlated in near infrared wavelength by FDTD. The sensitivity of the fiber was about $50 \mathrm{~nm} \times \mathrm{RIU}^{-1}$ and it had better refractive index detection. The tapered fiber can be applied to the bio-chemical sensors and physical deformation testing.
\end{abstract}

Keywords: tapered fiber; sensor; transmission spectrum

\section{Introduction}

There are many fiber optical sensors used in bio-chemical detection [1, 7-11, 16-18], environment monitoring [12-15] and physical tests [25]. The fiber optical sensors are used in transmission or reflection measurement setups. Depending on application requirements, the fiber optical sensor may measure transparent [19-21, 34] or turbid media [35, 36]. A simple fiber optical sensor construction requires standard fiber modification to enable environmental impact on light propagation parameters. Such modification may be performed by modification of fiber material parameters, for example long period gratings [14], or fiber shape modification by taper [16-18, 20] or D-shape [29] forming. The fiber sensor may be developed using experimental results [12-15] or mathematical modeling [16-18] approximation. The fiber optics may be made of polymer or silica. The forming of plastic fiber is relatively simple [31], while silica fibers shape modification requires advanced processing technology [32]. The processing technologies for tapered fiber are under development [33]. The tapered fiber diameter can achieve about $1 \mu \mathrm{m}$ and microfiber with diameter $500 \mathrm{~nm}$ has been

*E-mail: yanr163@163.com fabricated [20-23]. $90 \%$ of incident light can be coupled to the second microfiber connected parallel with first microfiber [19, 20]. Due to narrower width of the tapered fiber diameter, the tapered structure is more sensitive to refractive index change of surrounding environment [27-30]. A fiber optical sensor made by bending optical fibers can detect the refractive index of low-fat milk with excellent linearity between concentration and normalized response of the sensor [28]. A D-type sensor detects the liquid type by using intensity method for the sample [24-26, 29, 30]. The optical properties of the microstructure and thus those of the entire waveguide, depend on the optical properties of constituent materials as well as relative amount and arrangement of each.

In this paper, we have simulated transmission spectra for the tapered fibers without taper, one taper and two-tapered structures in near infrared wavelength by FDTD method. The transmission peaks and dips in the transmission spectra are sensitive to the taper number and taper parameters. The thickness sensitivity is defined as $\mathrm{k}=\Delta \lambda_{\text {peak }} / \Delta \mathrm{d}$ and is about $2.28 \mathrm{e}-3 \mu \mathrm{m}^{-1}$ for the tapered fiber [31]. The sensitivities of one taper and two-taper structures to the surrounding refractive index, simulated by FDTD, are different. The two-tapered fiber is more 
sensitive than the one-tapered fiber and its sensitivity is about $50 \mathrm{~nm} \times \mathrm{RIU}^{-1}$. The refractive index sensitivity is defined as $\mathrm{k}=\Delta \lambda_{\text {peak }} / \Delta \mathrm{n}$, and for the one-tapered fiber it is about $16.5 \mathrm{~nm} \mathrm{x} \mathrm{\textrm {RIU } ^ { - 1 }}$ [31]. Light self-focusing occurs at the outlet of the fiber and interference structures exist in the electric field distribution images for the tapered fibers. The electric field at the focus is about 20 times greater than the incident electric field and the focus diameter is about $500 \mathrm{~nm}$. Due to the smaller length of the fiber, there are interference structures in the electric field distribution visible in the calculated images for all types of analyzed heads presented in Fig. 1.

\section{Results and discussion}

The considered tapered fiber structure and fiber sensor principles are shown in Fig. 1. The diameter for the fiber core was $3 \mu \mathrm{m}$ and diameter for the fiber shell was about $6 \mu \mathrm{m}$. The refractive of the fiber core was 1.465 and 1.46 for the fiber shell. The length of the fiber was finite, which caused the electric field interference in the inlet and outlet. We fabricated the taper on the fiber using a special process technology and the taper structure similar to the common fiber. The core diameter of the taper was about $1.5 \mu \mathrm{m}$, the shell diameter was about $3 \mu \mathrm{m}$ and the deformation length was about $0.4 \mu \mathrm{m}$.

The tapered structure for the fiber is similar to that of the one-tapered fiber. The light emerging from the laser focuses at the fiber end-face by the objective and then propagates along the incident fiber. The light goes through the taper fiber sensor and emerges in the output fiber. Then the outlet light is detected by the detector. We have used the FDTD method to simulate the transmission spectra and electric field distribution. $\mathrm{SiO}_{2}$ with the refractive index of 1.465 was used as the core material. The light from the plane wave source propagates along the fiber and is diffracted at the taper position. The detector is positioned $5 \mathrm{~nm}$ away from the outlet face of the fiber.

We have simulated the transmission spectra, which are shown in Fig. 2, for the tapered fiber by the use of Finite-Difference-Time-Domain method
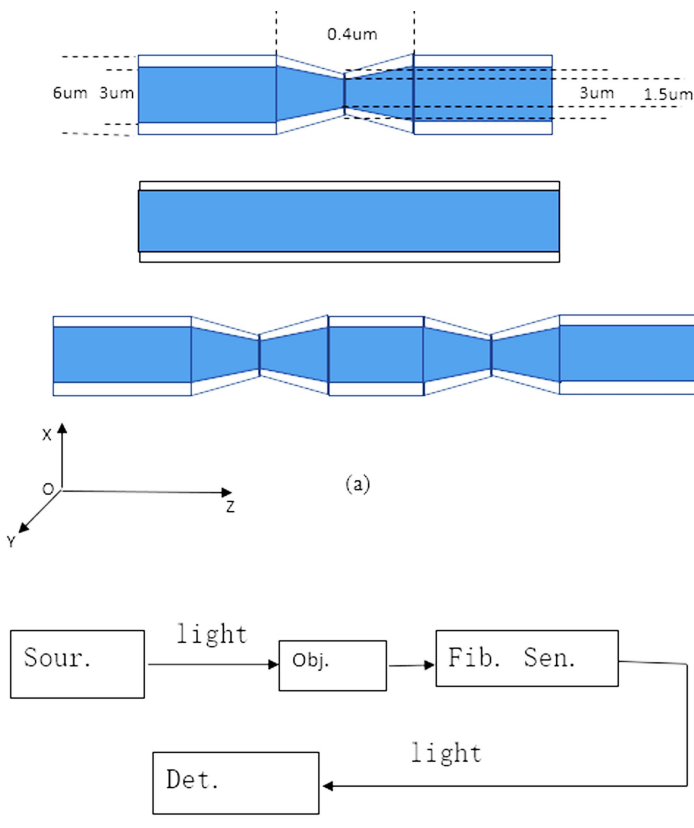

(b)

Fig. 1. (a) Tapered fiber structures; (b) detection scheme for the taper fiber sensor.

in the wavelength range of $1.3 \mu \mathrm{m}$ to $2.0 \mu \mathrm{m}$. We formed the taper at the fiber using a special process technology and the structure of the taper was similar to the fiber. The taper structure of the two-tapered fiber is similar to that of the onetapered fiber. Transmission peaks and transmission dips can be observed in the transmission spectra. The transmission amplitude, which is defined as the transmission electric field divided by incident electric field, can achieve about 0.24 for the tapered fiber. The transmission peak position for the non-tapered fiber shifted to the shorter wavelength in relation to one-tapered fiber and fiber. The transmission peak position was at $1.65515 \mu \mathrm{m}$ for the non-tapered fiber, $1.59102 \mu \mathrm{m}$ for the onetapered fiber and $1.5876 \mu \mathrm{m}$ for the two tapered fiber. The sensitivity for the tapered fiber was about $2.28 \mathrm{e}-3 \mathrm{~nm} \cdot \mu \mathrm{m}^{-1}$.

During the studies, one taper diameter of the two-tapered fiber was changed from $1.5 \mu \mathrm{m}$ to $1.0 \mu \mathrm{m}$ and the simulated transmission spectra are shown in Fig. 2b. There were also transmission peaks and dips in the wavelength range from $1.3 \mu \mathrm{m}$ to $2.0 \mu \mathrm{m}$. The transmission peak 

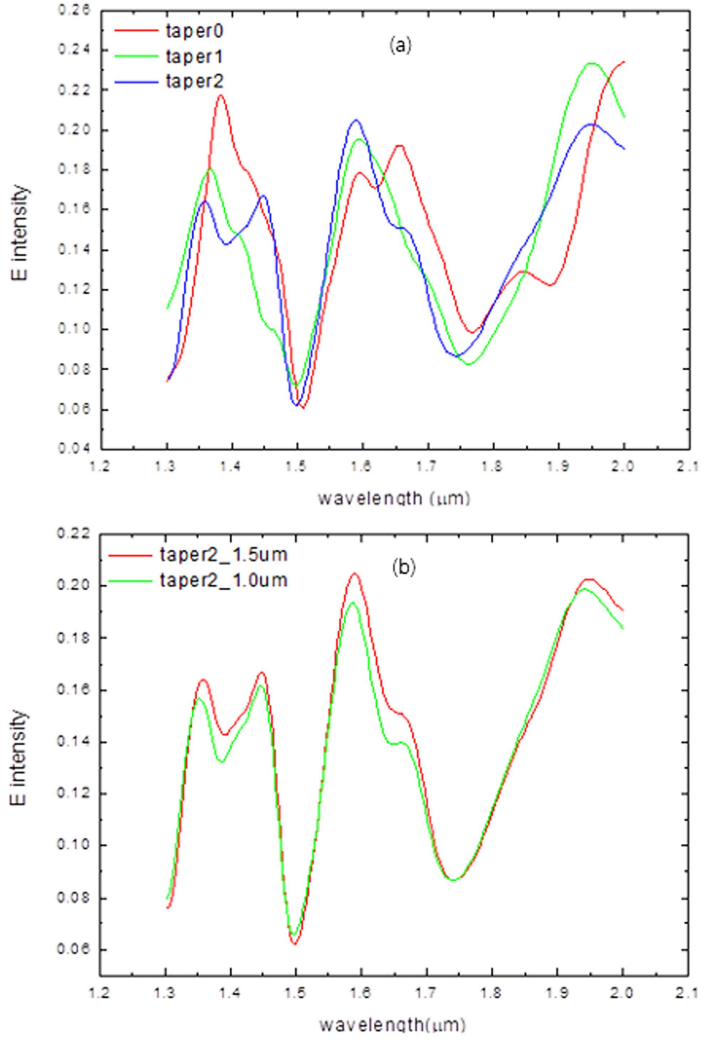

Fig. 2. (a) Simulated transmission spectra for the fiber without taper, one-tapered and two-tapered fibers; (b) simulated transmission spectra for the fibers with two tapers with the core thickness at the inlet of $1.5 \mu \mathrm{m}$ and $1.0 \mu \mathrm{m}$.

positions shifted to the shorter wavelength when the taper core diameter changed from $1.5 \mu \mathrm{m}$ to $1.0 \mu \mathrm{m}$. The transmission peak position was about $1.5876 \mu \mathrm{m}$ for the two-tapered fiber with deformation core diameter of $1.5 \mu \mathrm{m}$, and $1.5842 \mu \mathrm{m}$ for the two-tapered fiber with deformation core diameter $1.0 \mu \mathrm{m}$. The thickness sensitivity for the twotapered fiber on the thickness of the deformation was about $6.8 \mathrm{e}^{-3} \mathrm{~nm} \cdot \mu \mathrm{m}^{-1}[31]$.

The electric field distributions along $\mathrm{x}-\mathrm{z}$ plane for the fiber without taper, one taper, two tapers with deformation width of $1.5 \mu \mathrm{m}$ and two tapers with deformation width of $1.0 \mu \mathrm{m}$ are shown in Fig. 3. The light was incident in $\mathrm{z}$-axis and was polarized in $\mathrm{x}$-axis. The electric field distribution at the wavelength of $1.65515 \mu \mathrm{m}$ shown in Fig. 3a, corresponds to the transmission peak in the transmission spectrum for the fiber without taper. Smaller electric field localization and the electric field oscillation structures exist in the electric field distribution. The electric field is mainly localized at $500 \mathrm{~nm}$ and this localization results from electric field self-focus at the outlet of the fiber. The electric field can be enhanced about 18 times compared to the incident electric field.
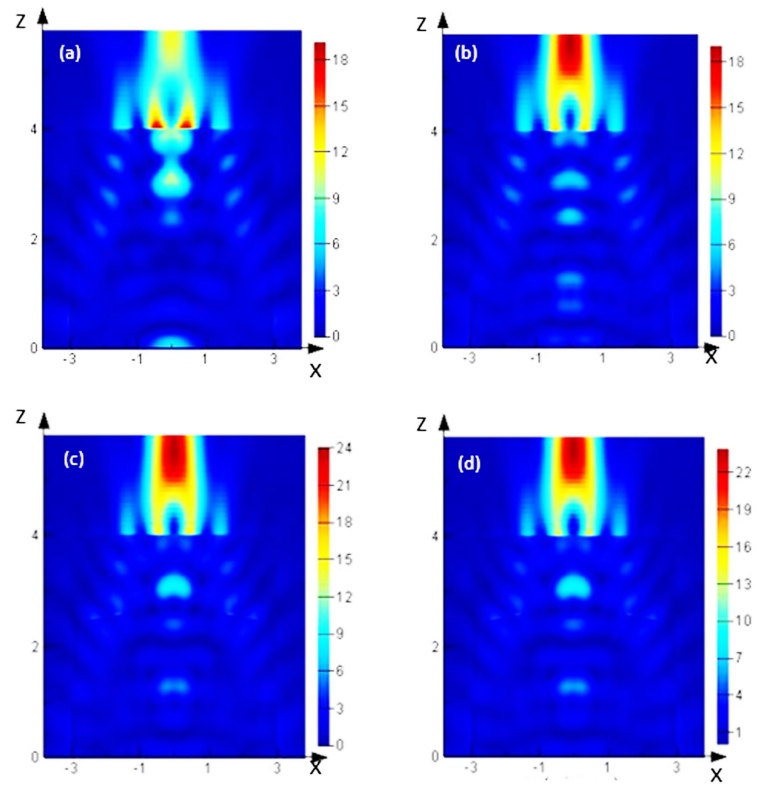

Fig. 3. Simulated electric field distribution for the fiber without taper, one-tapered and two-tapered fibers at wavelength of (a) $1.65515 \mu \mathrm{m}$; (b) $1.59102 \mu \mathrm{m}$; and (c) $1.5876 \mu \mathrm{m}$, respectively; (d) simulated electric field distribution for the two-tapered structures with the first taper width of $1.0 \mu \mathrm{m}$ at the wavelength $1.5842 \mu \mathrm{m}$.

The light can propagate along the fiber core and be reflected from the side wall of the fiber. In such case, there is an interference structure in the electric field profile shown in the near field images. The electric field distribution at the wavelength of $1.59102 \mu \mathrm{m}$ shown in Fig. 3b, corresponds to the transmission peak in the transmission spectrum of the one-tapered fiber. Compared with electric field profile for the fiber without taper, the interference structure for the one-tapered fiber is apparent and the electric field is enhanced 18 times in relation to the incident electric field. The electric field distribution at the wavelength of $1.5876 \mu \mathrm{m}$ 
is shown in Fig. 3c, which corresponds to the transmission peak for the fiber with deformation width $1.5 \mu \mathrm{m}$. The light propagates along the tapered fiber core and is reflected by the side wall. The interference structure existing in the electric field images results from the interaction reflected by the two tapers. The electric field is mainly localized at $500 \mathrm{~nm}$ at the outlet of the tapered fiber and is enhanced about 24 times in comparison to the incident electric field. The electric field distribution at the wavelength of $1.5842 \mu \mathrm{m}$ is shown in Fig. 3d, which corresponds to the transmission peak for the fiber with deformation width $1.0 \mu \mathrm{m}$. There was an interference structure in the electric field distribution, which was similar to the structure of the fiber with the deformation width of $1.5 \mu \mathrm{m}$. The electric field was enhanced about 22 times in relation to the incident electric field.

The transmission spectra of the one-tapered fiber with different taper shell refractive indices, simulated by using FDTD are shown in Fig. 4a. The taper structure is shown in Fig. 1, and the taper shell refractive index changed from 1.64 to 1.33. There are transmission peaks and dips in the transmission spectra and the transmission amplitude can achieve about 0.24 . Moreover, the transmission peaks shifted to the longer wavelength when the taper shell refractive index changed from 1.33 to 1.64 . The transmission peak position for the taper shell with refractive index 1.64 was at the wavelength of $1.591 \mu \mathrm{m}$, while it was at the wavelength $1.5842 \mu \mathrm{m}$ for the taper shell refractive index 1.33 . The sensitivity of the one-tapered fiber was about $22.25 \mathrm{~nm} \cdot \mathrm{RIU}^{-1}$ and is appropriate for bio-chemical detection. The electric field distribution along XOZ plane for the one-tapered fiber, was simulated by using FDTD. The electric field distributions at the wavelength $1.591 \mu \mathrm{m}$ for the taper shell refractive index of 1.64 and at the wavelength $1.5842 \mu \mathrm{m}$ for the taper shell refractive 1.33 are shown in Fig. $4 \mathrm{~b}$ and Fig. 4c. The electric field was incident in $\mathrm{Z}$-axis and polarized in $\mathrm{X}$-axis. Electric field localization and interference structure are shown in the electric field images. The electric field can be localized at $500 \mathrm{~nm}$ some microns apart from the outlet due to the self-focus effect
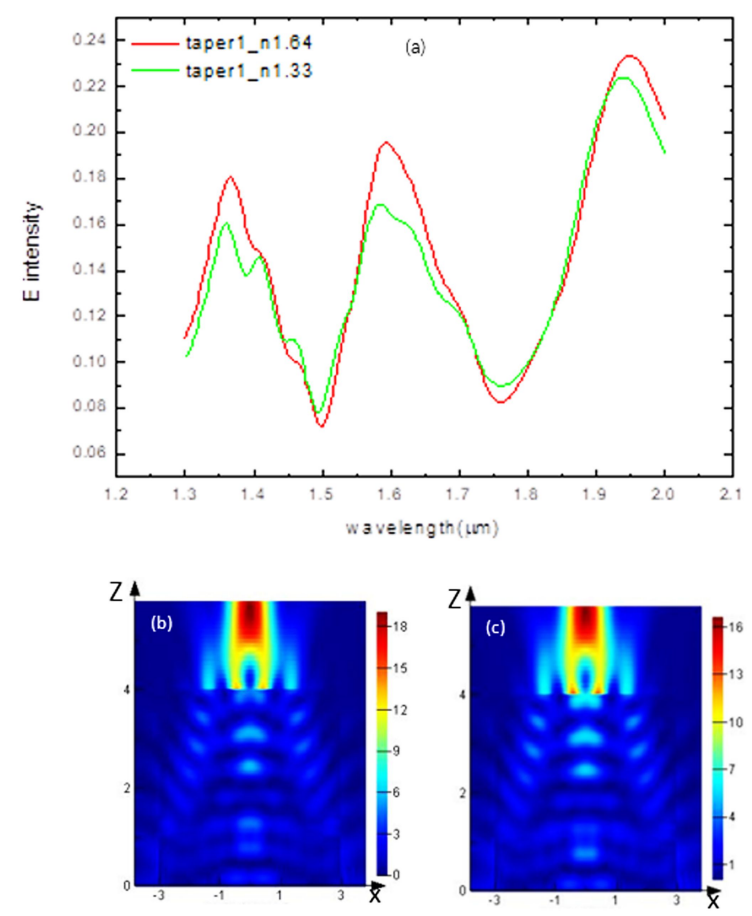

Fig. 4. (a) Transmission spectra of the fiber with one taper simulated by FDTD method where the refractive index of the taper shell structure changes from 1.64 to 1.33 ; (b) the simulated electric field distribution of the one-tapered fiber with the taper shell refractive index of 1.64 at the wavelength of $1.591 \mu \mathrm{m}$; (c) the simulated electric field distribution for the-one tapered fiber with the taper shell refractive index 1.33 at the wavelength of $1.5842 \mu \mathrm{m}$.

of the tapered fiber. The electric field is enhanced about 18 times in relation to the incident electric field for the taper shell with refractive index 1.64. In addition, the electric field can be enhanced about 16 times with respect to the incident electric field for the tapered shell with refractive index 1.33. The interference period was about $0.4 \mu \mathrm{m}$. The interference was more apparent for the taper shell refractive index 1.33 than that for the taper shell refractive index 1.64.The interference results from the interaction of the electric field reflected from the taper and the outlet.

The simulated transmission spectra for the fibers with different taper shell refractive indices obtained by the use of FDTD are shown in Fig. 5a. The transmission curve is smooth and reflects 
the profile of the transmission. The taper shell refractive changes from 1.0 to 1.64 and we have chosen the 1.64 and 1.33 transmission spectra depicted in the figure. There are transmission peaks and dips in the wavelength range from $1.3 \mu \mathrm{m}$ to $2.0 \mu \mathrm{m}$ in the transmission spectra, which resulted from the interference enhancement and destruction of the light reflected by the taper sidewalls.
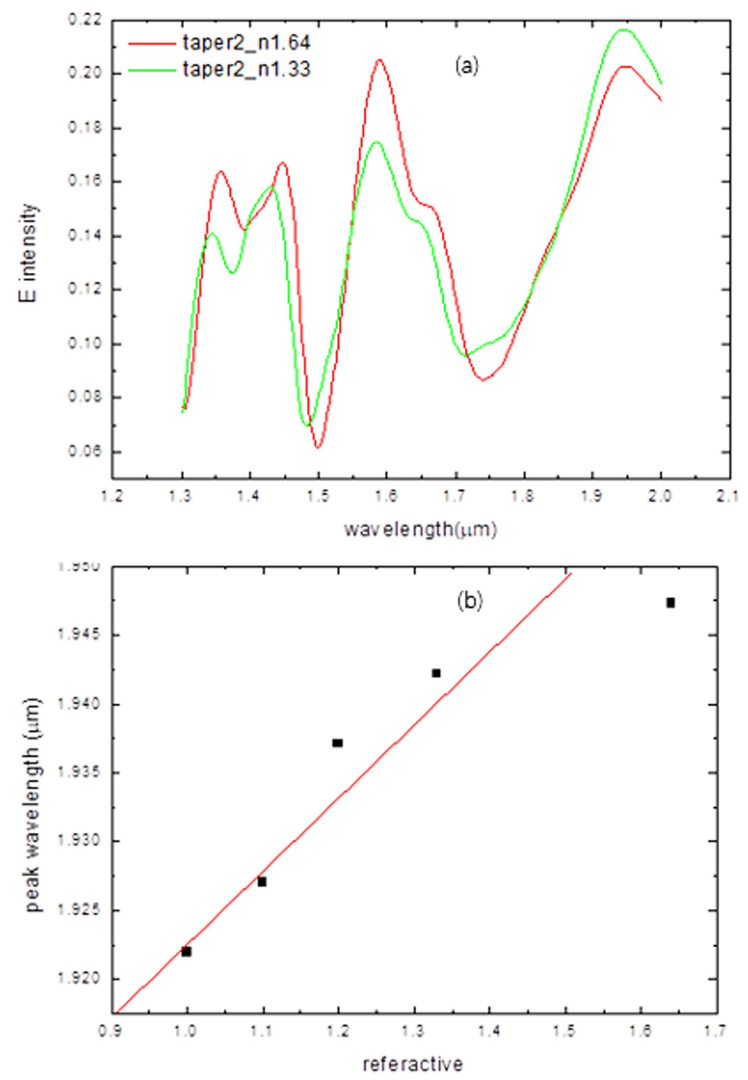

Fig. 5. (a) Simulated transmission spectra for the fiber with different shell refractive indices 1.64 and 1.33 , respectively; (b) the transmission peak positions for the two-tapered fiber with different refractive changing from 1.64 to 1.0 .

The transmission peaks shifted to the longer wavelength when the taper shell refractive index changed from 1.33 to 1.64 . The transmission peak for the taper shell refractive index 1.64 was at the wavelength $1.95245 \mu \mathrm{m}$ and for the taper shell refractive index 1.33, the peak occurred at the wavelength $1.94219 \mu \mathrm{m}$. The transmission amplitude for the fiber was about 0.20 and it was a little smaller
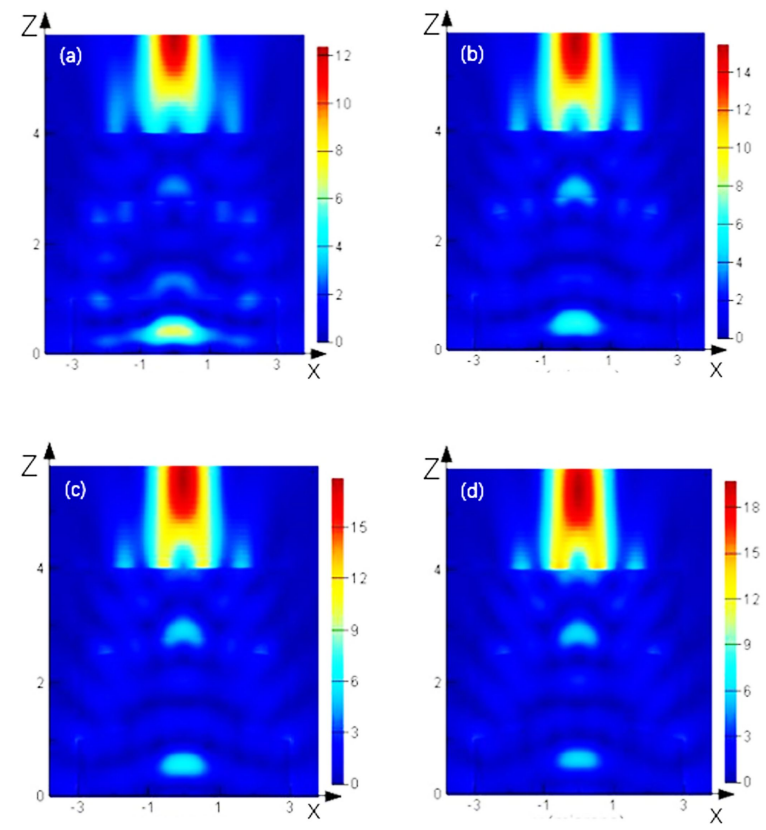

Fig. 6. Simulated electric field distribution for the fiber structures with the shell structure refractive index 1.0 at the wavelength $1.92199 \mu \mathrm{m}$ (a); 1.2 at the wavelength $1.9371 \mu \mathrm{m}$ (b); 1.33 at the wavelength $1.94219 \mu \mathrm{m}(\mathrm{c})$; and 1.64 at the wavelength $1.95245 \mu \mathrm{m}(\mathrm{d})$.

than that of the fiber itself. We have assessed the transmission peak positions shown in Fig. 5b based on the simulation results presented in Fig. 5a. The transmission peak positions shifted to longer wavelength as the taper shell refractive index gets larger. The sensor sensitivity is above $50 \mathrm{~nm} \times \mathrm{RIU}^{-1}$, which is suitable for the bio-chemical detection.

The electric field distributions in the $\mathrm{x}-\mathrm{z}$ plane for the fibers with different refractive indices of the taper shell are shown in Fig. 6: with refractive index 1.0 at the wavelength $1.92199 \mu \mathrm{m}-$ in Fig. 6a, with refractive index 1.2 at the wavelength $1.9371 \mu \mathrm{m}-$ in Fig. 6b, with refractive index 1.33 at the wavelength $1.94219 \mu \mathrm{m}-$ in Fig. 6c, and with the refractive index 1.64 at the wavelength $1.95245 \mu \mathrm{m}-$ in Fig. 6d. The interference structure in the electric field distribution and light focus occurs some microns apart from the fiber outlet. Better electric field localization and larger electric field enhancement existed in the electric field distribution images. The electric field was mainly 
localized at $500 \mathrm{~nm}$ and the electric field amplitude could achieve about 18 times greater value than the incident electric field. There was a little difference in the electric field distribution images for different refractive indices of the taper shell. The electric field amplitude gets larger when the taper shell refractive index was changed from 1.0 to 1.64 . The interference pattern was more apparent for the taper shell refractive index 1.64 than that for the taper shell refractive index 1.0. There were three interference stripes between the two tapers, which resulted from the two light beams interference reflected by the two tapers.

\section{Conclusions}

Transmission spectra of tapered fibers, simulated by the use of FDTD method in the wavelength range of $1.3 \mu \mathrm{m}$ to $2.0 \mu \mathrm{m}$ have been presented. The transmission peak positions are blue shifted when the taper number increased and the taper deformation was increased. The sensitivity to the deformation was about $2.28 \mathrm{e}^{-3} \mathrm{~nm} \cdot \mu \mathrm{m}^{-1}$, which is appropriate for physical tests. The sensitivity to the taper shell refractive index was about $50 \mathrm{~nm} \cdot \mathrm{RIU}^{-1}$ and was suitable for the bio-chemical detection. There was an interference structure in the electric field distribution and it was more apparent for larger taper shell refractive index and more fiber tapers.

\section{Acknowledgements}

This research was supported by the National Natural Science Foundation of China (No. 61178010 and No. 10805006), the Fundamental Research Funds for the Central Universities (No. bupt2010zx04), the National Program for Basic Research in China, Grant No. 2010 CB923202.

\section{References}

[1] Amanda J.H., Shengli Z., George C.S., Richard P.V.D., J. Phys. Chem. B, 108 (2004), 109.

[2] JiAngyan L., HAiQiang M., Optik, 124 (2013), 6419.

[3] Jiangyan L., Lin G., Jinxin F., Zhiyuan L., Chinese Phys. B, 22 (2013), 502.

[4] Jiangyan L., Kangsheng Q., Haiqiang M., Chinese Phys. B, 23 (2014), 429.

[5] Jiangyan L., Yilei H., JinXin F., ZhiyUan L., $J$. Appl. Phys., 107 (2001), 667.

[6] Jiangyan L., Zhiyuan L., Haifang Y., Aizi J., $J$. Appl. Phys., 104 (2008), 114303-1.
[7] Jorgenson R.C., YeE S.S., Sensor. Actuat. B-Chem., 12 (1993), 213.

[8] Jensen J.B., Hoiby P.E., Emiliyanov G., BAng O., Pedersen L.H., BJARKLEV A., Opt. Express, 13 (2005), 5883.

[9] Chinowsky T.M., Sensor. Actuat. B-Chem., 54 (1999), 89.

[10] Chinowsky T.M., YeE S.S., Sensor. Actuat. BChem., 51 (1998), 321.

[11] Johnston K.S., Sensor. Actuat. B-Chem., 54 (1999), 80.

[12] Krattiger B., Appl. Optics, 32 (1993), 956.

[13] Matsuura Y., Harrington J., J. Opt. Soc. Am. A, 14 (1997), 1255.

[14] Stellman C.M., Sensor. Actuat. B-Chem., 46 (1998), 56.

[15] Tarigan H.J., Anal. Chem., 68 (1996), 1752.

[16] Weigl B.H., Wolfbeis O.S., Anal. Chem., 66 (1994), 3323.

[17] Wolfbeis O.S., Trends Anal. Chem., 15 (1996), 225.

[18] Knight J.C., Birks T.A., Russell P.S.J., AtKin D.M., Opt. Lett., 21 (1996), 1547.

[19] Birks T.A., KNight J.C., Russel P.S.J., Opt. Lett., 22 (1997), 961.

[20] Eijkeleborg M.A.V., LARge M.C.J., Argvros A., Zagari J., Manos S., IsSA N.A., Assett B., Fleming S., MCPhedran R.C., Desterke C.M., Ninorovici N.A.P., Opt. Express, 9 (2001), 319.

[21] Monro T.M., West Y.D., HewaK D.W., BrodERICK N.G.R., RICHARDSON D.J., Electron Lett., 23 (2000), 1998.

[22] Newby K., Reichert W.M., Andrade J.D., BenNER R.E., Appl. Optics, 23 (1984), 1812.

[23] Culshaw B., Muhammad F., Stewart G., MurRAY S., Pinchbeck D., Norris J., CASSidy S., Wilkinson M., Williams D., CRisP I., EWYK R.V., MCGHEe A., Electron Lett., 28 (1992), 2232.

[24] Hale Z.M., Payne F.P., Proc. IEE. Coll. Fib. Opt. Sen. Tech. Dig. Num., 128 (1992, 81.

[25] Hoo Y.L., Jin W., Ho H.L., WANG D.N., Windeler R.S., Opt. Eng., 41 (2002), 8.

[26] Jensen J.B., Pedersen L.H., Hoiby P.E., Nielsen L.B., HANSEN T.P., FOLKENBERG J.R., RiISHEDE J., NoordegraAF D., Nielsen K., CARlsen A., BJARKLEV A., Opt. Lett., 29 (2004), 1974.

[27] Wang H.P., Wang Y.C., Leung P.T., Thin solid films, 425 (2003), 135.

[28] Saracoglu O.G., Hayber S.E., Sensors-Basel, 16 (2016), 2094.

[29] Borecki M., Proc. SPIE. Opt. Fib.-Appl., 5952 (2005), 5952181.

[30] Iadicicco A., Campopiano S., Cutolo A., GiorDAno M., Cusano A., IEEE Photonic Tech. L., 17 (2005), 1250.

[31] Kruszewski J., Borecki M., Beblowska M., Proc. SPIE., 5576 (2004), 234. 
[32] Paipulas D., Mikutis M., Sirutkaitis V., JuOdKAZIS S., Proc. SPIE., 8786 (2013), 87860D.

[33] Xiaomei S., Abraham H.D., Proc. SPIE., 2391 (1995), 512.

[34] Zhao Y., Liao Y.B., Sensor. Actuat. B-Chem., 86 (2002), 63.

[35] BORECKI M., DOROZ P., PRUS P., PsACZOLKOWSKi P., SZMIDT J., KorWIN-PAWLOWSKI M.L., Frydrych J., Kocjubinski A., DUK M., Int. J. Adv. Sys. Meas., 7 (2014), 57.
[36] Ilev I.K., Wayant R.W., Byrnes K.R., Anders J.J., Opt. Lett., 27 (2002), 1695.
Received 2016-12-10

Accepted 2018-11-12 